\title{
The application of the electrical automation in power system
}

\author{
Laiyun Hou \\ Department of Electric Power Engineering, North China Electric Power University, 689 Huadian Road, \\ Baoding, 071003, China \\ houlaiyun123@163.com
}

Keywords: electrical automation, power system, computer technology, PLC technology

\begin{abstract}
On the basis of introducing the development of electrical automation, this paper detailedly analyzes the application of computer technology and PLC technology, which are specific technologies of electrical automation used in the power system. The purpose of this paper is to make people have a deeper understanding of the application of electrical automation technology in power system and provide reference for the improvement, improvement, innovation and development of electrical automation technology.
\end{abstract}

\section{Summary of electrical automation}

The electrical automation system is mainly composed of three parts: a component that is designed to receive signals transmitted by the relevant equipment, a component that effectively processes the received signal, and a component that outputs signals related to the electrical equipment. Execution terminals (power plants, substations, etc.) and dispatch and control terminals are two very important components of the electrical automation system. However, the automation system has completely different work contents in the two components of the power system. They complete the work contents of each part in sequence, so that the effective analysis and overall control of the corresponding data of the power system can be realized. The work mainly includes the following three aspects: On the one hand, it collects the operating data and related parameters of the electric terminal (substation, power plant, etc.) system and realizes this part of the work by scheduling; on the other hand, Of the operating conditions of the effective analysis and accurate judgments, which control side to perform and complete; the final one is the control of the power system to analyze, determine and form a planning directive, the operation of the relevant electrical equipment and its Parameter adjustment and other commands to the executive end (substations, power plants, etc.), in order to timely and smoothly complete the measurement and control tasks.

\section{Electrical automation development process}

Since the concept of electrical automation was proposed, its development has mainly gone through the following stages.

\subsection{Semi-controlled thyristor was replaced by full-controlled power electronic switch}

Thyristor appeared from the beginning as the first generation of power electronic devices, so far in our country is still widely used in DC and AC drive control system, until the second generation of fully controlled devices, three generations of composite devices until the last Four generations of power electronics, the drive power, over-voltage over-current protection, current detection and even the temperature of the automatic control and so on as a whole.

\subsection{Inverter circuit from low frequency to high frequency}

High-frequency inverter circuit does not hinder the inverter to increase the operating frequency at the same time the switching losses can be minimized, thus reducing the size of the inverter, effectively reducing costs, but also can make the inverter at higher power Under the integration, we can see the development of DC inverter circuit is very promising. 


\subsection{Universal inverter into large-scale use}

General Inverter generally refers to the batch, serialization, in the market demand for larger medium and small power inverter. From the inverter products to analyze, U / F controller from the initial ordinary functional type into a high-functional type, until the later high-performance vector control type, until now to power semiconductor IGBT devices, its unique and reliable Sex, operability and easy maintenance features such as single-chip control technology has also been a corresponding increase.

\subsection{The progress of power system automation is promoted by computer and electronic technology}

In the 1980s, the rapid development and widespread application of SCM technology enabled the power system automation equipment to be fully updated. Domestic industrial computers and the introduction of PC technology in the power plant monitoring system, dispatching automation power system, integrated substation automation has played a crucial role. On this basis, the developed application software can fully realize the operation of the power system for real-time data collection, aggregation, classification, analysis, display, printing and completion. However, during this period there are still some problems with the electrical automation. For example, devices from different manufacturers can not be interconnected. Because the point-to-point star connection is generally used between the equipment and the computer, the real-time performance of the system is not good, which directly leads to Equipment configuration flexibility is poor; the entire system in the function, system structure, communication protocols, lack of appropriate industry standards.

\section{Application of Electrical Automation in Power System}

\subsection{Computer technology in power system automation applications}

Computer control technology plays a crucial role in the power system. This is because with the rapid development of computer technology, power system and other important aspects as well as transmission, power generation, distribution and transformation sectors need the support of computer technology, which will make the power system automation technology has been rapidly developed.

3.1.1 Application in power plants

The power plant is an important part of the power system. The automation level of the power plant directly determines the efficiency of the power generation operation. Both the wind power generation and the thermal power generation require the use of an automatic control system. At present, the automation technology applied in wind power generation is mainly used for controlling the control of the blade turning angle and the monitoring, protection and control so that the wind power generation equipment can automatically turn to the wind and control the stable cleaning of the power generation equipment. The main automation technology of hydroelectric power mainly controls the movement potential of water. The automation technology is mainly used in information monitoring, protection system and power generation control system, which can be applied to measurement units, voltage regulation and normal hydroelectric power generation. Mainly used in thermal power generation for coal fuel control, relay protection control and fault handling, you can also use information management, data monitoring, and automated operation and control and so on.

\subsubsection{Application in substation}

The process of applying electrical automation technology in the substation application process mainly refers to the substation using the high-tech computer technology to achieve the operation based on the combination of related application information processing technology, electrical system automation control technology and related equipment information transmission technology Automate the effects and process of managing automation effects.

\subsubsection{Application in Power Network Dispatch Control}

In electrical engineering, grid dispatching technology refers to dynamically balancing generation and consumption of electricity in the grid by controlling related servers. The application of this electrical automation technology is mainly achieved through the following three aspects: First, the 
safety, stability and high efficiency of the power grid in its operation are mainly achieved through its economic dispatch technology; second, the electrical automation Automation of system-related load forecasting is mainly achieved by timely and accurate monitoring and analysis of the corresponding electrical equipment operation data and power production process data; Third, the accuracy of finding, determining and eliminating the fault of the relevant electrical network system of electrical engineering electrical automation system Sexuality, timeliness and efficiency are achieved through the display of relevant electrical equipment data.

\subsubsection{Application in distribution automation}

The current distribution scale is still small, the use of automation technology can meet the needs of small-scale power distribution equipment management, data transfer and other needs, through computer technology to provide users with efficient services to achieve efficient and reliable operation of the power system to achieve the goal. At present, power distribution automation has been linked with artificial intelligence theory to realize large-scale centralized control supported by optical fiber communication. This is of great significance for effective exchange of master and substation data and formation of an efficient power distribution system.

\subsection{Application of PLC Technology in Power System Automation}

PLC is a combination of computer technology and relay contact control technology. The memory adopts programmable logic control, arithmetic operation and record operation in its internal storage. This technology is a programmable logic controller system designed for use in industrial environments. This technology has been widely used in power system automation in recent years, which solves the defects of low reliability, complicated wiring, poor flexibility and high energy consumption in the traditional control system.

\subsubsection{PLC technology data processing}

PLC is a combination of computer technology and relay contact control technology. The memory adopts programmable logic control, arithmetic operation and record operation in its internal storage. This technology is a programmable logic controller system designed for use in industrial environments. This technology has been widely used in power system automation in recent years, which solves the defects of low reliability, complicated wiring, poor flexibility and high energy consumption in the traditional control system.

\subsubsection{Closed loop process control of PLC technology}

Closed-loop process control refers to the pressure, temperature, flow and other continuous changes in the analog closed-loop control, PLC through the analog I/O module for analog closed loop PID control, and to achieve digital and analog D/A, A/D conversion. You can use a dedicated PID module, PID subroutine can also be used to achieve.

\subsubsection{PLC technology switch control}

Auxiliary systems within the thermal power generation system control of the process control and digital control of two kinds of switch. Many industries also use PLC for switch control, such as machine electrical control, motor control and elevator operation control, automotive assembly line and beer filling production lines. PLC technology output and input signals are on / off switch signal. The most widely used control in industrial control is the logic control of the switch. Control output, the number of input points can be achieved through the expansion, from a dozen to tens of thousands of points, unlimited.

\subsubsection{PLC control of the order of technology}

With the gradual improvement of national energy saving and emission reduction requirements and the deepening of reform, the auxiliary systems of large-scale thermal power enterprises have been upgraded from the original relay controllers to PLC control systems in recent years, reducing resource consumption and improving efficiency in the production process, Has become the ultimate goal of the management of all enterprises. Therefore, with the advancement of science and technology, the power plants also put forward higher requirements on the level of automatic control of auxiliary workshops of similar enterprises. However, the PLC control system can not only control a certain process flow 
through the information module alone but also connect with the communication bus Coordinate the entire factory production.

\section{Summary}

Electrical automation system with real-time, high level of automation, management features such as strong, can improve power supply reliability and power quality, improve customer service, with significant economic advantages and good overall social benefits. Therefore, we must achieve the sustainable development of electrical automation technology through scientific development.

\section{References}

[1]. Aimin Li. The development trend of electrical automation and its application in power system. Science \& technology information.Vol.23(2012)No.27,p.131

[2]. Yong Deng. The Application and Development of Electrical Automation in Power System. Electronic Technology \& Software Engineering. Vol.47(2016)No.23,p124

[3]. T. B. Lo, M. F. Mendes, H. A. L. Samaniego and R. S. d. Oliveira, "Cloud IEC 61850: Architecture and Integration of Electrical Automation Systems," 2014 Brazilian Symposium on Computing Systems Engineering, Manaus, 2014, pp. 13-18.

[4]. Jie Ren. Application of Electrical Automation Technology in Power System.Theoretical Research. Vol.22(2012),p.31-32

[5]. S. Deda, R. Greul, J. Ornig, N. Stefanov, O. König and G. Prochart, "Automated Hardwarein-the-loop testing for high voltage/power system "AVL E-STORAGE BTE"," IECON 2016 42nd Annual Conference of the IEEE Industrial Electronics Society, Florence, 2016, pp. 6699-6704. 